\title{
Facial cutaneous sinuses of dental origin - a diagnostic challenge
}

IN BRIEF
- Depicts the clinical presentation of facial
cutaneous sinus tracts.
- Explains how to correctly diagnose facial
cutaneous sinuses.
- Describes the management of facial
cutaneous sinuses.

\author{
S. Sammut, ${ }^{* 1}$ N. Malden² and V. Lopes ${ }^{3}$
}

\begin{abstract}
It is common for practitioners to misdiagnose the cause of facial cutaneous sinus tracts, failing to recognise that many have an odontogenic cause. Chronic infection around the apex of a dental root can drain to the mouth or less commonly to the skin via a sinus tract. Dental symptoms are not always present and this confuses the clinical picture further. Failure to identify an odontogenic cause may result in unnecessary and ineffective treatment. Elimination of dental infection via tooth extraction or root canal treatment leads to resolution of the cutaneous sinus. We present a series of cutaneous draining sinuses of dental origin that resolved rapidly following dental treatment and hope to highlight the importance of including odontogenic infection in the differential diagnosis of such a lesion in the head and neck.
\end{abstract}

\section{INTRODUCTION}

Cutaneous sinus tracts of dental origin continue to be a diagnostic challenge. ${ }^{1,2}$ They are frequently misdiagnosed as lesions of non-odontogenic origin by physicians and surgeons alike, which leads to incorrect and unnecessary treatment. ${ }^{2,3}$ The lesion may persist for long periods ${ }^{4}$ before the correct diagnosis is made and the odontogenic source treated appropriately. ${ }^{5,6}$

A sinus tract is a communication between an enclosed area of inflammation/ infection to an epithelial surface or body cavity. ${ }^{7}$ The most common cause of cutaneous sinus tracts in the face and neck is the result of chronically draining dental infection. ${ }^{4,8}$ Such chronic draining sinuses should signal the need for a thorough dental evaluation. As a result of misdiagnosis, patients may be subjected to unnecessary and ineffective treatment such as repeated courses of antibiotics, multiple biopsies, surgical excision of the cutaneous

\footnotetext{
${ }^{1}$ Speciality Registrar in Oral Surgery, ${ }^{2}$ Consultant in Oral Surgery, ${ }^{3}$ Consultant in Oral and Maxillofacial Surgery, Edinburgh Dental Institute, Combined Department of Oral and Maxillofacial Surgery and Oral Medicine, Lauriston Building, Lauriston Place, Edinburgh, EH3 9HA ${ }^{*}$ Correspondence to: Stephanie Sammut

Email: stefsammut@gmail.com; Tel: 00447986621882
}

\section{Refereed Paper}

Accepted 25 July 2013

DOI: 10.1038/sj.bdj.2013.1141

${ }^{\bullet}$ British Dental Journal 2013; 215: 555-558 lesion and in some cases even radiotherapy. ${ }^{9}$ This brings about only a temporary resolution of the sinus and is followed inevitably by recurrence. ${ }^{9}$ Once the source of odontogenic infection is eliminated, healing of the sinus is expected within 5 to 14 days. $^{2,3}$

\section{NATURAL HISTORY OF THE CONDITION}

Infection around the root apex of a tooth arises following pulpal necrosis, which is usually the result of dental caries or traumatic injury. ${ }^{9}$ The inflammatory process that starts in the dental pulp spreads to the periodontal ligament and into the surrounding bone ${ }^{9}$ to initiate a periapical dental abscess. ${ }^{8}$ This progresses slowly, resorbing bone and spreading towards the cortical plate along the path of least resistance. ${ }^{4,8}$ Once the abscess perforates through the cortical bone it may spread into a fascial space, localise into an abscess, or may develop into a cellulitis. ${ }^{9}$ The development of a sinus tract from an abscess drains the suppuration, most commonly into the mouth, and less commonly onto the skin. ${ }^{6}$ If the infection tracks out of the jaw above the buccinator muscle attachment in the maxilla or below the mentalis, mylohyoid or buccinator attachments in the mandible, the sinus drains extra-orally. If the perforation of the cortical plate is below the muscle attachments in the maxilla and above the muscle attachments in the mandible, the sinus is more likely to drain intra-orally (Fig.1)., ${ }^{2,410}$ The point of drainage depends in part on the length of the root and the position of the apex relative to the muscular attachments. Some authors ${ }^{4,11}$ claim that extra-oral 
sinuses are more common in children and adolescents because the teeth are not yet fully erupted and the alveolar process is not fully developed and so roots are more deeply seated. However, most case reports available are predominantly of adults ${ }^{10}$ and thus do not support this view.

As a result of drainage, chronic low grade infection is not always associated with a history of acute pain ${ }^{9}$ and patients seldom relate the cutaneous lesion with a dental cause.

\section{CLINICAL PRESENTATION}

The extra-oral draining sinus typically presents as an erythematous, smooth, non-tender nodule with crusting, which drains pus periodically. 'Dimpling' or retraction below the normal skin surface is characteristic. ${ }^{9}$ It is usually possible to palpate a cord-like tract that attaches to the underlying alveolar bone in the area of the suspected tooth.

Symptoms from the teeth may only present in $50 \%$ of patients ${ }^{8}$ and this may be why patients often initially seek help from a physician. In addition, the cutaneous draining sinus may be some distance from the primary odontogenic cause ${ }^{4}$ and these two factors combined may contribute to a delay in diagnosis. The involved tooth is always non vital but may not always be tender to percussion. ${ }^{7}$

Mandibular teeth are implicated over maxillary teeth in a ratio of $4: 1^{8,12}$ with $50 \%$ of mandibular sinuses emanating from lower incisors or canines. It is not surprising therefore that the commonest cutaneous sinus tract is seen in the chin or submental region. ${ }^{8}$ Premolars will commonly point to the submandibular region while lower molars can point on submandibular skin or to the cheek. Maxillary incisors may point to the floor of the nose, ${ }^{13}$ while canine teeth will commonly point to below the inner canthus of the eye. Maxillary premolars and molars may point to the cheek. ${ }^{4}$

\section{DIAGNOSIS}

To reach a correct diagnosis, the attending clinician must look carefully for a potential odontogenic infection. A thorough history may pick up the incidence of any traumatic injuries to the teeth, recently or in the past. ${ }^{8,9}$ Sometimes patients are able to recall a history of toothache

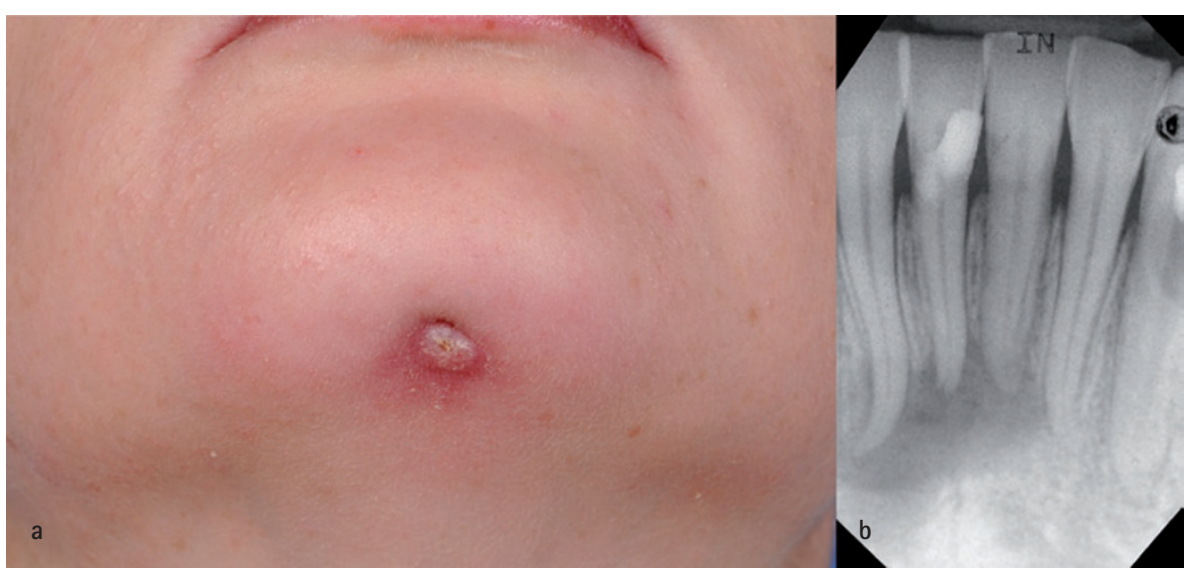

Fig. 2 Case 1: (a) Extraoral view of midline draining sinus on the chin; (b) Periapical radiograph showing rarefaction at the root apices

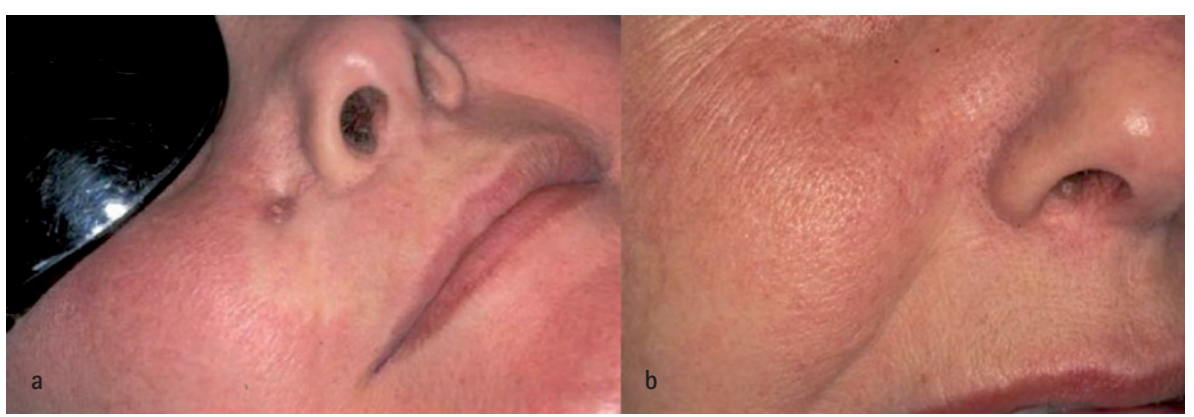

Fig. 3 Case 2: (a) Extraoral view showing draining sinus in right nasolabial area; (b) Extra-oral view six months post-operatively, showing complete resolution of the sinus following apical surgery

before the development of the sinus. ${ }^{7}$ Intraoral examination should identify any discoloured or heavily restored teeth. Pulp testing and intraoral radiographs will help to identify the culpable tooth. A radiopaque marker (gutta-percha point) may be inserted into the sinus before radiographic exposure to determine the source. ${ }^{3,8}$

\section{DIFFERENTIAL DIAGNOSIS}

Dental infection is the most prevalent aetiology for cutaneous sinus tracts in the face and neck region and should be considered first in a differential diagnosis. ${ }^{4}$ Other causes include: osteomyelitis, actinomycosis, foreign body, local skin infection, pyogenic granuloma, salivary gland and duct fistula, suppurative lymphadenitis and neoplasm among others.

\section{TREATMENT}

The dental infection must be managed, either with endodontic treatment or extraction. ${ }^{9}$ Surgical excision of the cutaneous sinus is not necessary as spontaneous closure of the tract is expected within 5 to 14 days of dental treatment. ${ }^{2}$
Healing occurs by secondary intention and occasionally a residual scar may persist. ${ }^{10}$ In these cases surgery may be indicated to improve aesthetics.

What follows is a case series of patients who presented to the Combined Department of Oral and Maxillofacial Surgery at the Edinburgh Dental Institute with extra-oral draining sinuses of dental origin.

\section{CASE SERIES}

\section{Case one}

A 29-year-old female patient with Crohn's disease was referred to the Edinburgh Dental Institute by her gastrointestinal consultant with an extra-oral draining sinus on her chin. This had been present for a month and had been treated with a course of amoxicillin and flucloxacillin, but failed to respond.

The patient had widespread dental caries, which was considered attributable to the quantity of fentanyl lozenges she consumed per day. They are known to have a high sugar content.

Clinical examination revealed a draining sinus in the midline of the chin that was oozing pus (Fig. 2a). Several mandibular 


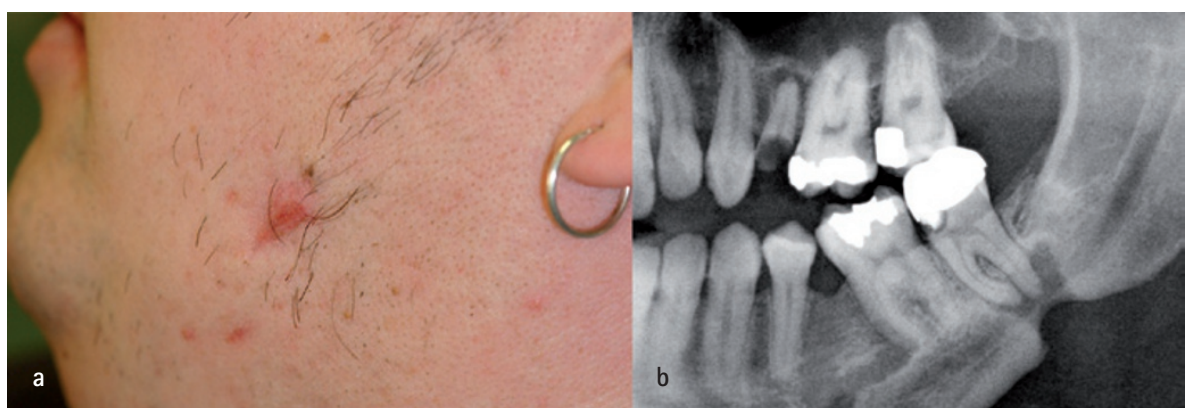

Fig. 4 Case 3: (a) Clinical picture showing scar from previous excision in left cheek; (b) Orthopantomograph showing bone loss associated with heavily restored lower left posterior molar

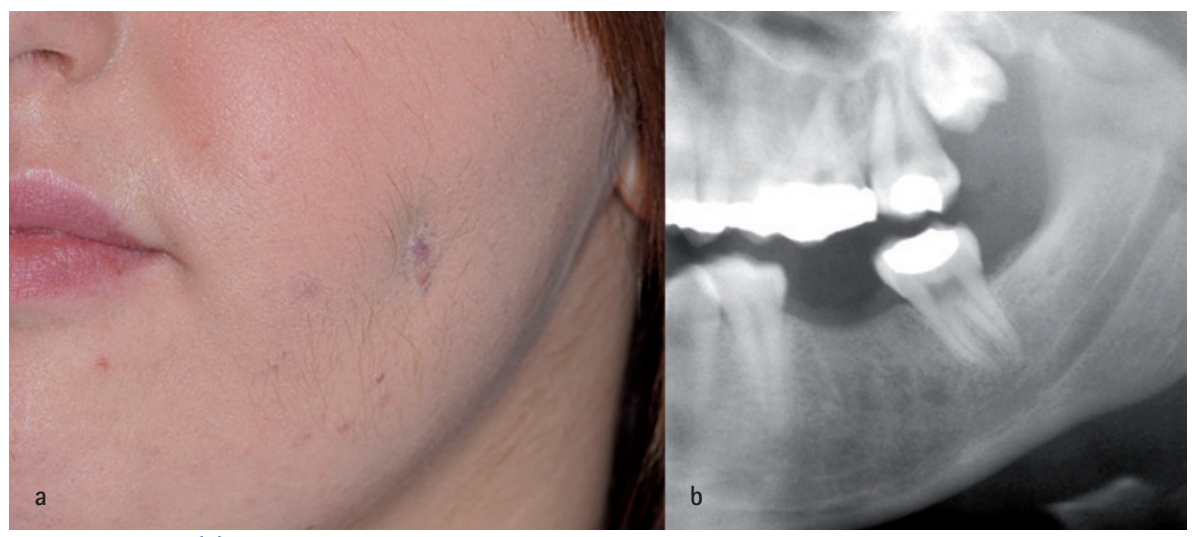

Fig. 5 Case 5: (a) Extraoral view showing tethered draining sinus in the left cheek associated with heavily restored and non-vital maxillary 1st and 2nd molar teeth; (b) Orthopantomograph showing periapical rarefaction around the roots of upper left first and second molar teeth.

anterior teeth were carious and an intraoral radiograph demonstrated an area of periapical infection associated with the lower incisor teeth (Fig. 2b). The teeth were root-treated by her dental practitioner. Complete healing was noted three months post-treatment.

\section{Case two}

A 50-year-old woman was referred to the Combined Department of Oral and Maxillofacial surgery with a facial sinus in the right nasolabial area (Fig. 3a). She had no complaints apart from the unsightly appearance of the sinus. Clinical examination revealed a small intraoral swelling in the right maxillary canine region with a palpable fibrous tract that connected with the skin lesion. The canine was heavily restored with a post-crown and was already root-treated. Intra-oral radiographs revealed a periapical area of infection around the apex of this tooth. She was listed for apical surgery of the canine and retrograde root filling. Re-root treatment was considered unfeasible due to the post-retained restoration. At her post-operative review two weeks later, the facial sinus had dried up but there was a persistent scar. On her review six months later the scar had resolved spontaneously and the patient was discharged (Fig. 3b).

\section{Case three}

A fit and well 29-year-old male was referred to the Combined Department of Oral and Maxillofacial surgery with an external draining sinus on his left cheek close to the lower border of the mandible. The sinus had been surgically excised under local anaesthetic in the past by a plastic surgeon, but it had recurred. Clinical examination revealed a heavily restored dentition. The lower left second molar was tender to percussion and restored with a porcelain-metal crown. Radiographs revealed a sizeable periapical area of infection with bone loss of the lower margin of the mandible (Fig. 4). The tooth was extracted under local anaesthetic and the sinus resolved one month later. The patient was left with a minimal scar, however he preferred not to have this surgically corrected.

\section{Case four}

A fit and well 60-year-old man was referred to the Combined Department of Oral and
Maxillofacial Surgery with an extra-oral draining sinus in the right submandibular area. This oozed pus intermittently and had been present for a few months. The gentleman had no recollection of dental pain. Clinical examination revealed two remaining roots in the lower arch. Radiographs revealed periapical infection around both these roots. These were extracted under local anaesthetic. On his post-operative review four weeks later the sinus had dried up completely and there was minimal scarring.

\section{Case five}

A fit and well 21-year-old woman was referred to the Combined Department of Oral and Maxillofacial Surgery with a persistent, relatively painless, extra-oral facial sinus in the left cheek (Fig. 5a). This had been present for four years. During this time, she was prescribed several courses of antibiotics and had undergone surgical excision of the sinus. None of these measures were able to eradicate the lesion. On questioning, the patient recalled an episode of dental pain before the development of the sinus.

Clinical examination revealed a large fibrous scar on the left cheek. Intra-orally the upper left first and second molars were heavily restored and an intra-oral draining sinus was visible next to these teeth. Radiographs revealed a periapical area of infection associated with the first molar (Fig. 5b). This was extracted under local anaesthetic. At the end of the six month follow up period the scar in her left cheek was almost imperceptible.

\section{Discussion}

Cutaneous facial sinuses may be difficult to diagnose: patients may not always have or recall dental symptoms and the cutaneous sinus may develop at a distance from the origin of infection. ${ }^{14}$ Misdiagnosis of these lesions results in unnecessary treatment including antibiotic therapy, surgical excision ${ }^{2}$ and even radiotherapy. ${ }^{15}$ Antibiotic therapy will bring a temporary resolution of drainage and apparent healing. However, if the source of infection is not eliminated the sinus tract will recur shortly. ${ }^{2}$

It is estimated that half of the patients with extra-oral fistulae are submitted to multiple dermatological surgical 
operations and long-term antibiotic therapy before the correct diagnosis is made. ${ }^{14}$ It is important that physicians have an awareness that a cutaneous lesion of the face and neck could be of dental origin ${ }^{4}$ and should always seek a dental opinion. ${ }^{14}$

\section{CONCLUSION}

A dental aetiology must always be considered for any cutaneous sinus tract in the head or neck. Elimination of the dental source of infection results in resolution of the sinus tract without the need for surgical excision and long-term unacceptable aesthetic results.
Thanks to Professor Crispian Scully CBE for permission to reproduce the image of draining pathways and to the medical illustrations department at St. John's Hospital, Livingston for assistance with images.

1. Cohenca N, Karni S, Rotstein I. Extraoral sinus tract misdiagnosed as an endodontic lesion. J Endod 2003; 29: 841-843.

2. Johnson B R, Remeikis N A, Van Cura J E. Diagnosis and treatment of cutaneous facial sinus tracts of dental origin. J Am Dent Assoc 1999;130: 832-836.

3. McWalter G M, Alexander J B, del Rio C E, Knott J W. Cutaneous sinus tracts of dental aetiology. Oral Surg Oral Med Oral Pathol 1988; 66: 608-614.

4. Lewin-Epstein J, Taicher S, Azaz B. Cutaneous sinus tracts of dental origin. Arch Dermato/ 1978; 114: $1158-1161$

5. Gorsky M, Kaffe I, Tamse A. A draining sinus tract of the chin. Report of a case. Oral Surg Oral Med Oral Pathol 1978; 46: 583-587.

6. Soares J A de Carvalho F B, Pappen FG et $a$. Conservative treatment of patients with periapical lesions associated with extraoral sinus tracts. Aust Endod J 2007; 33: 131-135.
7. Mukerji $R$, Jones D C. Facial sinus of dental origin: a case report. Dent Update 2002; 29: 170-171.

8. Cioffi G A, Terezhalmy G T, Parlette H L. Cutaneous draining sinus tract: an odontogenic aetiology. J Am Acad Dermatol 1986; 14: 94-100.

9. Mittal N, Gupta P. Management of extra oral sinus cases: a clinical dilemma. J Endod 2004; 30: 541-547.

10. Spear K L, Sheridan P J, Perry H O. Sinus tracts to the chin and jaw of dental origin. J Am Acad Dermatol 1983: 8: 486-492.

11. Azaz B, Taicher S. Facial sinus tracts of dental origin in children. ASDC J Dent Child 1976; 43: 167-171.

12. Hodges T P, Cohen D A, Deck D. Odontogenic sinus tracts. Am Fam Physician 1989; 40: 113-116.

13. Strader R J, Seda H J. Periapical abscess with intranasal fistula. Oral Surg Oral Med Oral Pathol 1971; 32: 881-884

14. Pasternak-Junior B, Teixeira C S, Silva-Sousa Y T, SousaNeto M D. Diagnosis and treatment of odontogenic cutaneous sinus tracts of endodontic origin: three case studies. Int Endod J 2009; 42: 271-276.

15. Tidwell $E$, Jenkins J D, Ellis C D, Hutson B, Cederberg $R$ A. Cutaneous odontogenic sinus tract to the chin: a case report. Int Endod J 1997; 30: 352-355. 